\title{
Full Salpeter Equation with Confining Interactions: Analytic Stability Proof
}

\author{
Wolfgang Lucha* \\ Institute for High Energy Physics, Austrian Academy of Sciences, Nikolsdorfergasse 18, A-1050 \\ Vienna, Austria \\ E-mail: Wolfgang.Lucha@oeaw.ac.at
}

\begin{abstract}
The most popular 3-dimensional reduction of the Bethe-Salpeter formalism for the description of bound states within quantum field theory is the Salpeter equation, found as the instantaneous limit of the Bethe-Salpeter framework if allowing, in addition, for free propagation of the bound-state constituents. Unfortunately, depending on the chosen Lorentz nature of the Bethe-Salpeter kernel, which encodes all interactions between the bound-state constituents, supposedly stable results of Salpeter's equation with confining interactions exhibit (un-)expected instabilities, probably related to Klein's paradox. Clearly, bound states may be regarded as stable if, for appropriate interactions, their energy eigenvalues or, in the center-of-momentum system, their mass eigenvalues belong to a real and discrete (part of the) spectrum that is bounded from below. Discreteness means either that eigenvalues and a possible continuous spectrum are disjoint or that the spectrum is purely discrete. Some general features of the eigenvalue spectra of any Salpeter equation, common to all solutions, are well-established: Since Salpeter's equation proves to be of the same algebraic structure as the random-phase-approximation equation familiar in nuclear physics all bound-state masses squared are real. Of course, this implies neither that the mass spectrum itself is necessarily real nor, even in those cases where it may be shown to be real, that it is also bounded from below. Direct inspection reveals that, for a large class of sufficiently reasonable Bethe-Salpeter kernels-which includes all interactions of phenomenological relevance in QCD—, the spectrum of mass eigenvalues consists, in the complex bound-state mass plane, at most of real pairs of opposite sign and imaginary points. In general, if the Lorentz structure of a confining kernel is a mixture of time-component vector and scalar stability is achieved only if the Lorentz structure is predominantly a time-component vector. For time-component vector harmonic-oscillator kernels, a straightforward stability proof is given.
\end{abstract}

8th Conference Quark Confinement and the Hadron Spectrum

September 1-6, 2008

Mainz, Germany

${ }^{*}$ Speaker. 
Building on experience gained in earlier investigations focused to the simpler reduced Salpeter equation [1,2] and its improvement [3, 4] obtained by inclusion of full (dressed) propagators for the bound-state constituents [5, 6], a rigorous proof of the full-Salpeter solutions' stability for confining (such as harmonic-oscillator) interactions of time-component Lorentz-vector nature is constructed. For interactions described, in configuration space, by central potentials of harmonic-oscillator form, $V(\mathbf{x})=a \mathbf{x}^{2}, a \neq 0$, the integral equation representing the Salpeter equation simplifies to a system of second-order homogeneous linear ordinary differential equations. Instabilities should appear first in pseudoscalar bound states, and this problem is most severe and restrictive for massless constituents. Such setting allows for a thorough spectral analysis of the problem: The interaction of the fermionic bound-state constituents is of time-component Lorentz-vector nature if both their couplings to some effective Lorentz-scalar potential are represented by the Dirac matrix $\gamma$. In this case, bound states exist for $a>0$; the squares of their masses are determined by the eigenvalues of the product $O \equiv B A$ of two positive self-adjoint (Schrödinger) operators $A$ and $B$ on the Hilbert space $L^{2}\left(\mathbb{R}^{3}\right)$, defined by

$$
A \equiv-a \Delta+2|\mathbf{x}|=A^{\dagger} \geq 0, \quad B \equiv-a \Delta+2|\mathbf{x}|+\frac{2 a}{\mathbf{x}^{2}}=B^{\dagger} \geq 0, \quad \Delta \equiv \nabla \cdot \nabla .
$$

Using the (unique) positive self-adjoint square root $A^{1 / 2}=\left(A^{1 / 2}\right)^{\dagger} \geq 0$ of $A$, the eigenvalue problem for $O$ is found to be equivalent to that for the positive self-adjoint operator $Q \equiv A^{1 / 2} B A^{1 / 2}=Q^{\dagger} \geq 0$ while the operator inequality $A \leq B$, clearly satisfied by $A$ and $B$, translates into the relation $A^{2} \leq Q$. Because its potential, $2|\mathbf{x}| / a$, is bounded from below and rises to infinity for $|\mathbf{x}| \rightarrow \infty$, the operator $A$ and thus also the operator $A^{2}$ have purely discrete spectrum consisting of real eigenvalues which rise to infinity. Then, applying the minimum-maximum principle to $A^{2} \leq Q$ reveals that our bound-state masses squared and, since $Q \geq 0$, also all the masses themselves are part of a real discrete spectrum.

\section{References}

[1] Z.-F. Li, W. Lucha, and F. F. Schöberl, Stability in the instantaneous Bethe-Salpeter formalism: harmonic-oscillator reduced Salpeter equation, Phys. Rev. D 76 (2007) 125028 [arXiv:0707.3202 [hep-ph]].

[2] W. Lucha and F. F. Schöberl, Stability of Salpeter solutions, in QCD@Work 2007 - International Workshop on Quantum Chromodynamics: Theory and Experiment, edited by P. Colangelo et al., AIP Conf. Proc. (AIP, Melville, New York, 2007), Vol. 964, p. 318 [arXiv: 0707.1440 [hep-ph] ].

[3] W. Lucha and F. F. Schöberl, Instantaneous Bethe-Salpeter equation with exact propagators, J. Phys. G: Nucl. Part. Phys. 31 (2005) 1133 [hep-th/0507281].

[4] W. Lucha and F. F. Schöberl, Generalized instantaneous Bethe-Salpeter equation and exact quark propagators, in Quark Confinement and the Hadron Spectrum VII: $7^{\text {th }}$ Conference on Quark Confinement and the Hadron Spectrum - QCHS7, edited by J. E. F. T. Ribeiro, AIP Conf. Proc. (AIP, Melville, New York, 2007), Vol. 892, p. 524 [hep-ph/0610016].

[5] W. Lucha and F. F. Schöberl, Stability of the solutions of instantaneous Bethe-Salpeter equations with confining interactions, in XII International Conference on Hadron Spectroscopy - Hadron 07, edited by L. Benussi et al., Frascati Phys. Ser. (INFN Laboratori Nazionali di Frascati, 2007), Vol. 46, p. 1539 [arXiv:0711.1736 [hep-ph] ].

[6] Z.-F. Li, W. Lucha, and F. F. Schöberl, Stability in the instantaneous Bethe-Salpeter formalism: a reduced exact-propagator bound-state equation with harmonic interaction, J. Phys. G: Nucl. Part. Phys. 35 (2008) 115002 [arXiv: 0712.2947 [hep-ph] ]. 